\title{
Generic exponential fringe model for alleviating phase error in phase measuring profilometry
}

\author{
Chao Chen ${ }^{1}$, Nan Gao ${ }^{1}$, Xiangjun Wang ${ }^{2}$, Zonghua Zhang ${ }^{1,3^{*}}$, Feng Gao ${ }^{3}$, Xiangqian \\ Jiang $^{3}$ \\ ${ }^{1}$ School of Mechanical Engineering, Hebei University of Technology, 300130 Tianjin, \\ China. \\ ${ }^{2}$ State Key Laboratory of Precision Measuring Technology and Instruments, Tianjin \\ University, Tianjin 300072, China. \\ ${ }^{3}$ Centre for Precision Technologies, University of Huddersfield, Huddersfield, HD1 \\ 3DH, UK \\ *zhzhangtju@ hotmail.com, zhzhang@ @ebut.edu.cn
}

\begin{abstract}
Phase measuring profilometry (PMP) is susceptible to phase error caused by gamma distortion that leads to the captured fringe patterns deviating from ideal sinusoidal waveforms. Existing phase-error compensation methods are generally complex and require significant computational resources for implementation. This paper proposes a generic exponential fringe model expressed as an exponential function of the generated fringe patterns. Based on this model, a straightforward gamma correction method is presented to alleviate phase error without the need for any estimation of nonlinear coefficients or complex calibration. Experimental results demonstrate that the proposed method improves the quality of measurements by suppressing phase error.
\end{abstract}

Keywords: phase-error compensation, gamma correction, exponential fringe model, phase measuring profilometry

\section{Introduction}

Phase measuring profilometry (PMP) is an important technique in three-dimensional (3D) measurement technology owing to its advantages like non-contact operation, full-field acquisition, high resolution, and high-speed data processing [1-2]. However, PMP is prone to phase errors due to gamma distortion, which causes the captured fringe patterns to deviate from ideal sinusoidal waveforms. The phase error due to a nonlinear response of a projector-camera system is undesirable and results in a poor measurement accuracy [3]. Many phase-error compensation methods have been proposed to overcome this nonlinear response. These methods can be categorized in two main types: a) calibration based methods and b) defocusing based methods [4]. As the name suggests, calibration based compensation methods depend on a calibration procedure to quantify the nonlinearity of system response by using techniques like response curve fitting [5], phase benchmark building [6, 7], and gamma model derivation [8-12]. Huang et al. [5] pre-coded the projection grating to reduce measurement error caused by the nonlinear gamma. Zhang et al. [6] proposed a gamma-correction method based on a look-up table (LUT) constructed through statistical analysis of the fringe images. This technique was able to detect the phase-error distribution caused by gamma in order to compute the 
correct phase. An iterative method proposed by Pan et al. [7] was able to simplify phase error to just one order. Hoang et al. [8] proposed a method to decrease the intensity nonlinearity by combining a large-step phase-shifting algorithm with a gamma-correction method. The method proposed by Liu et al. [9] involved a model of the harmonic coefficients of the gamma value. Li et al. [10] developed a theoretical model of the gamma-distorted fringe image to calculate the gamma factor. Zhang et al. [11] combined the methods proposed by Liu [9] and Li [10], and developed a universal gamma model to eliminate phase error. Cai et al. [12] introduced two methods to realize robust active and passive phase-error compensation that could adapt to dynamic measurement conditions. Their methods were based on a universal phase-error model which was suitable for an arbitrary phase-shifting step. Although the phase error caused by the nonlinear response can be compensated effectively with these methods, the calibration procedure requires some prior knowledge of the system nonlinear response obtained from projection and capturing of numerous images. Moreover, due to the sequential nature of the calibration and measurement processes, the phase error can increase making the procedure counter-productive.

The second type of error-compensation methods rely on defocusing, which acts as a low-pass filter to eliminate high-frequency harmonics [13-16]. Lei et al. [13] observed that sinusoidal fringe maps can be obtained from the defocusing of binary patterns (DBP). They concluded that the error involved in the focusing of sinusoidal patterns is larger than DBP methods. Since the DBP method is not sensitive to the nonlinear gamma, it removes the requirement of gamma correction. This approach only uses single-frequency fringe patterns, hence it is not suitable for steep gradients or discontinuous surfaces. To overcome this problem, Huang et al. [14] introduced the multi-frequency heterodyne phase shifts into the DBP method. Wang et al. [15] presented an optimal pulse-width modulation method for wide fringe pattern generation. Xu et al. [16] proposed a method to reduce phase error by combining phase-shifting algorithms with the gamma problem. This method can be used for dynamic 3D shape measurements as there is no requirement for synchronization between the camera and projector. In spite of the benefits of the defocusing approach, precise control over the degree of defocusing still remains as a challenging task [17-18].

There are other methods for decreasing the gamma effect without correcting phase or intensity errors. A direct and effective method [19] that does not introduce any additional calculation in the 3D shape determination used encoded initial fringe patterns before projection. Although, this method deals with the gamma problem at the source, the gamma value is typically not a fixed constant. Liu et al. [20] proposed a method based on inverse function shift estimation to detect the spatial shift of the distorted phase caused by object height. This spatial shift can reconstruct an object depth and is independent of projector's gamma distortion. However, the correspondence relationship between shift and depth needs to be determined beforehand.

Therefore, existing phase-error compensation methods or gamma correction methods require some prior or post experimental information such as gamma value, or the correspondence relationship between the input and output light intensities. The acquisition procedure of this data is often iterative and complicated, and requires significant computational resources. In this paper, a generic exponential fringe model is derived from an optical perspective. Based on this model, a direct and simple gamma correction method is proposed for alleviating phase error without requiring any prior and post setup information.

The remainder of the paper is organized as follow. Section 2 introduces the theory of the proposed exponential fringe projection method. Section 3 and 4 shows the simulations and the actual 
experimental results, respectively. The conclusions and future remarks are given in Section 5.

\section{Theory}

\subsection{Traditional fringe projection method}

In fringe projection profilometry, a traditional phase-shifting fringe sequence is generated by a cosine function. Assuming that the projector's input sinusoidal fringe patterns generated by a computer, the intensity can be expresses as follows,

$$
I_{n}\left(u^{p}, v^{p}\right)=A\left(u^{p}, v^{p}\right)+B\left(u^{p}, v^{p}\right) \cos \left[\varphi\left(u^{p}, v^{p}\right)+\delta_{n}\right]
$$

where, $A\left(u^{p}, v^{p}\right), B\left(u^{p}, v^{p}\right)$, and $\varphi\left(u^{p}, v^{p}\right)$ are the intensity bias, intensity modulation, and solved phase, respectively, at each projector pixel coordinate $\left(u^{p}, v^{p}\right), \delta_{n}=2(n-1) \pi / N$ is the phase shift amount, $n=1$, $2, \ldots, N$ is the image index, and $N$ is the total number of fringe patterns. Output intensity of the fringe patterns can be expressed as,

$$
I_{n}\left(u^{p}, v^{p}\right)=\left\{A\left(u^{p}, v^{p}\right)+B\left(u^{p}, v^{p}\right) \cos \left[\varphi\left(u^{p}, v^{p}\right)+\delta_{n}\right]\right\}^{\gamma}
$$

where, $\gamma$ is the gamma value. After the projector projects the fringe patterns onto a surface, the reflected fringe patterns are captured by a camera from a different viewpoint. Assuming that the camera has a linear response to the intensity and the ambient light is neglected, the intensity of the captured gamma-distorted fringe pattern can be expressed as,

$$
I_{n}\left(u^{c}, v^{c}\right)=\left\{A\left(u^{c}, v^{c}\right)+B\left(u^{c}, v^{c}\right) \cos \left[\varphi\left(u^{c}, v^{c}\right)+\delta_{n}\right]\right\}^{\gamma}
$$

where, $A\left(u^{c}, v^{c}\right), B\left(u^{c}, v^{c}\right)$, and $\varphi\left(u^{c}, v^{c}\right)$ are the intensity bias, intensity modulation, and solved phase, respectively, at each camera pixel coordinate $\left(u^{c}, v^{c}\right)$. The general approach for a phase-shifting method involves using a least-square method to fit measured phase-shifted values with a cosine function. The wrapped phase can be calculated from the following,

$$
\varphi\left(u^{c}, v^{c}\right)=\arctan \frac{-\sum_{n=1}^{N} I_{n}\left(u^{c}, v^{c}\right) \sin \delta_{n}}{\sum_{n=1}^{N} I_{n}\left(u^{c}, v^{c}\right) \cos \delta_{n}}
$$

\subsection{Exponential fringe projection method}

To avoid gamma effect in the traditional fringe projection methods, this paper proposes a new fringe projection method called exponential fringe projection. The intensity of the exponential fringe image can be expressed as,

$$
I_{n}\left(u^{p}, v^{p}\right)=e^{A\left(u^{p}, v^{p}\right)+B\left(u^{p}, v^{p}\right) \cos \left[\varphi\left(u^{p}, v^{p}\right)+\delta_{n}\right]}
$$

In order to bring out the difference between the exponential and traditional fringe patterns, fringe patterns having two fringe periods based on Eq. (1) and (5) are demonstrated in Fig. 1(a) and Fig. 1(b), respectively. The corresponding profiles of normalized intensity along the middle row direction are illustrated in Fig. 1(c). Assuming that $I_{n}$ satisfies $I_{\min } \leq e^{A\left(u^{p}, v^{p}\right)+B\left(u^{p}, v^{p}\right) \cos \left[\varphi\left(u^{p}, v^{p}\right)+\delta_{n}\right]} \leq I_{\max }$, the following equations hold, 


$$
\begin{aligned}
& 0 \leq A\left(u^{p}, v^{p}\right) \leq \frac{\ln I_{\text {max }}+\ln I_{\text {min }}}{2} \\
& 0 \leq B\left(u^{p}, v^{p}\right) \leq \frac{\ln I_{\text {max }}-\ln I_{\text {min }}}{2}
\end{aligned}
$$

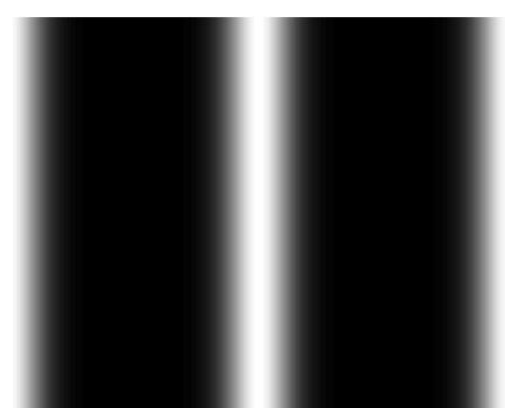

(a)

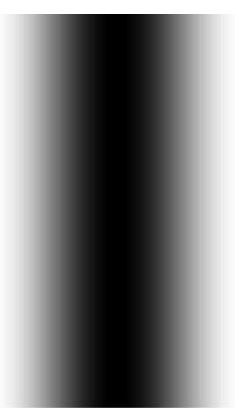

(b)

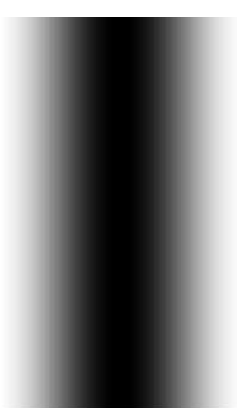

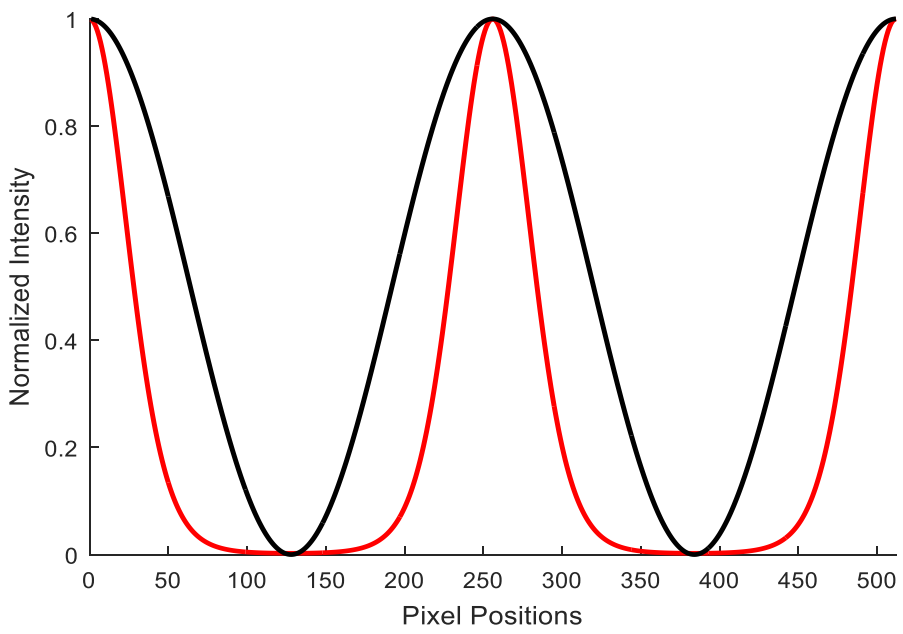

(c)

Figure 1 Intensity of fringe patterns. (a) Exponential pattern; (b)

Traditional pattern; (c) Profiles of normalized intensity along the middle row direction.

Equation (6) is used to limit the range of the background intensity and modulation intensity of the generated fringe pattern. Therefore, optimal values for $A$ and $B$ can be selected by using Eq. (6) when generating exponential fringe patterns. A projected exponential fringe pattern has the expression of Eq. (5) because of the nonlinear response of the projector. Without consideration the effect of ambient light, the captured gamma-distorted fringe pattern on an object surface by the camera can be expressed as Eq. (7) if the used camera has a linear response. The captured gamma-distorted fringe pattern can be expressed as,

$$
I_{n}\left(u^{c}, v^{c}\right)=e^{\left\{A\left(u^{c}, v^{c}\right)+B\left(u^{c}, v^{c}\right) \cos \left[\varphi\left(u^{c}, v^{c}\right)+\delta_{n}\right]\right\} \gamma}
$$

Taking the logarithm on the both sides of the Eq. (7) yields,

$$
\ln I_{n}\left(u^{c}, v^{c}\right)=\gamma\left\{A\left(u^{c}, v^{c}\right)+B\left(u^{c}, v^{c}\right) \cos \left[\varphi\left(u^{c}, v^{c}\right)+\delta_{n}\right]\right\}
$$

Eq. (8) can be derived as,

$$
\ln I_{n}\left(u^{c}, v^{c}\right)=\gamma\left\{A\left(u^{c}, v^{c}\right)+B\left(u^{c}, v^{c}\right) \cos \left[\varphi\left(u^{c}, v^{c}\right)\right] \cos \delta_{n}-B\left(u^{c}, v^{c}\right) \sin \left[\varphi\left(u^{c}, v^{c}\right)\right] \sin \delta_{n}\right\}
$$


Assuming that $p\left(u^{c}, v^{c}\right)=B\left(u^{c}, v^{c}\right) \cos \left[\varphi\left(u^{c}, v^{c}\right)\right]$ and $q\left(u^{c}, v^{c}\right)=B\left(u^{c}, v^{c}\right) \sin \left[\varphi\left(u^{c}, v^{c}\right)\right]$, the least-squares difference $E\left(u^{c}, v^{c}\right)$ between the theoretical pattern and measured pattern for all $N$ fringe patterns can be written as:

$$
E\left(u^{c}, v^{c}\right)=\sum_{n=1}^{N}\left\{\gamma A\left(u^{c}, v^{c}\right)+\gamma p\left(u^{c}, v^{c}\right) \cos \delta_{n}-\gamma q\left(u^{c}, v^{c}\right) \sin \delta_{n}-\ln I_{n}^{\prime}\left(u^{c}, v^{c}\right)\right\}^{2}
$$

To minimize $E\left(u^{c}, v^{c}\right)$, the partial derivative of Eq. (10) with respect to $p\left(u^{c}, v^{c}\right), q\left(u^{c}, v^{c}\right)$ and $A\left(u^{c}\right.$, $\left.v^{c}\right)$ is computed and equated to zero, resulting the following criteria,

$$
\begin{array}{lll}
\frac{\partial E\left(u^{c}, v^{c}\right)}{\partial p\left(u^{c}, v^{c}\right)}=0 & \frac{\partial E\left(u^{c}, v^{c}\right)}{\partial q\left(u^{c}, v^{c}\right)}=0 & \frac{\partial E\left(u^{c}, v^{c}\right)}{\partial A\left(u^{c}, v^{c}\right)}=0
\end{array}
$$

Solving Eq. (11) yields,

$$
X=\frac{1}{\gamma} \cdot U^{-1} Q
$$

where,

$$
U=\left[\begin{array}{ccc}
\sum_{n=1}^{N} \cos ^{2} \delta_{n} & -\sum_{n=1}^{N} \sin \delta_{n} \cos \delta_{n} & \sum_{n=1}^{N} \cos \delta_{n} \\
-\sum_{n=1}^{N} \sin \delta_{n} \cos \delta_{n} & \sum_{n=1}^{N} \sin ^{2} \delta_{n} & -\sum_{n=1}^{N} \sin \delta_{n} \\
\sum_{n=1}^{N} \cos \delta_{n} & -\sum_{n=1}^{N} \sin \delta_{n} & N
\end{array}\right]
$$$$
X=\left[p\left(u^{c}, v^{c}\right) \quad q\left(u^{c}, v^{c}\right) \quad A\left(u^{c}, v^{c}\right)\right]^{T}
$$$$
Q=\left[\sum_{n=1}^{N}\left(\cos \delta_{n}\right) \ln I_{n}^{\prime}\left(u^{c}, v^{c}\right) \quad-\sum_{n=1}^{N}\left(\sin \delta_{n}\right) \ln I_{n}^{\prime}\left(u^{c}, v^{c}\right) \quad \sum_{n=1}^{N} \ln I_{n}^{\prime}\left(u^{c}, v^{c}\right)\right]^{T}
$$

The wrapped phase can be determined from the following equation,

$$
\varphi\left(u^{c}, v^{c}\right)=\arctan \frac{-\sum_{n=1}^{N}\left(\sin \delta_{n}\right) \ln I_{n}^{\prime}\left(u^{c}, v^{c}\right)}{\sum_{n=1}^{N}\left(\cos \delta_{n}\right) \ln I_{n}^{\prime}\left(u^{c}, v^{c}\right)}
$$

It is clear that the proposed exponential fringe model is independent on the gamma value. Therefore, it provides a direct yet effective solution, and does not require any additional calculations in the 3D shape measurement.

\section{Simulations}

To validate the efficacy of the proposed method, a series of simulated fringe patterns $(512 \times 512$ pixels, and four-step phase-shifting) were projected on a white flat board and the images were subsequently captured. Two kinds of fringe patterns were tested: the traditional sinusoidal and the proposed exponential fringe patterns.

Both the phase-shifting fringe patterns were encoded with $\gamma=3.2$ without random noise. The simulation results of the traditional sinusoidal and the proposed exponential fringe patterns are shown in Figure 2(a) - (d) and Figure 2(e) - (h), respectively. The two captured fringe patterns are shown in Figure 2(a) and Figure 2(e), and their profiles along the middle row direction are illustrated in Figure 
2(b) and Figure 2(f). For the traditional sinusoidal fringe patterns, the corresponding wrapped phase map is influenced by the higher-order harmonics, as shown in Figure 2(c) and Figure 2(d).

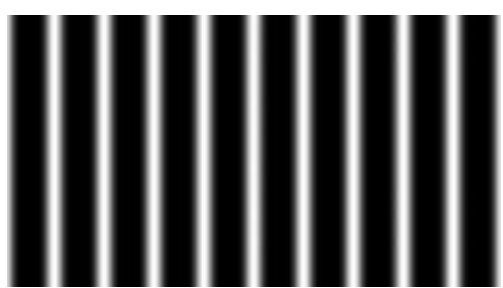

(a)

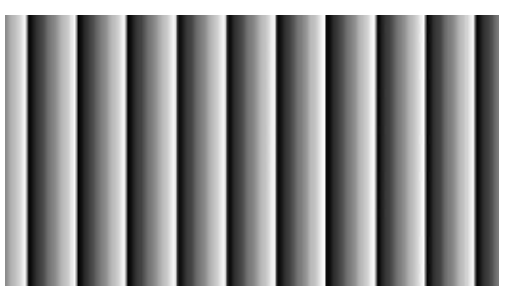

(c)

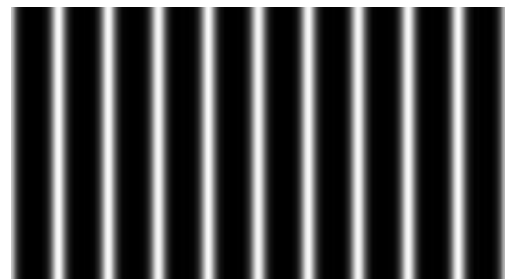

(e)

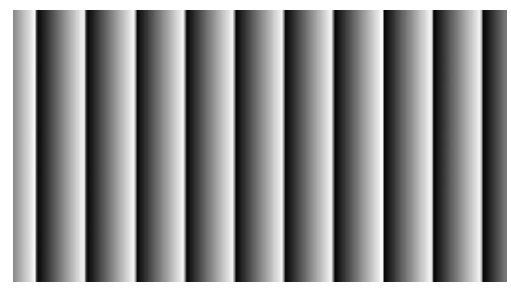

(g)

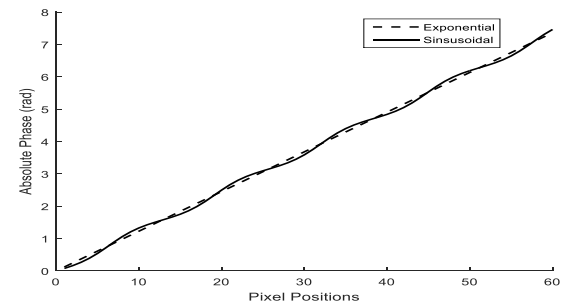

(i)

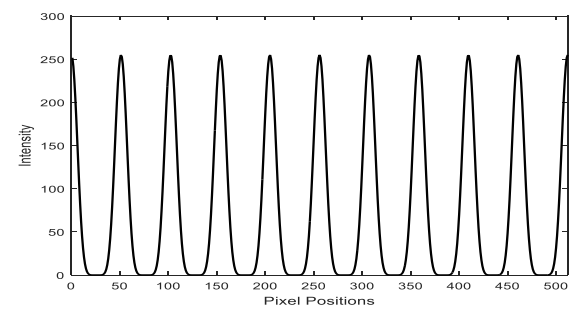

(b)

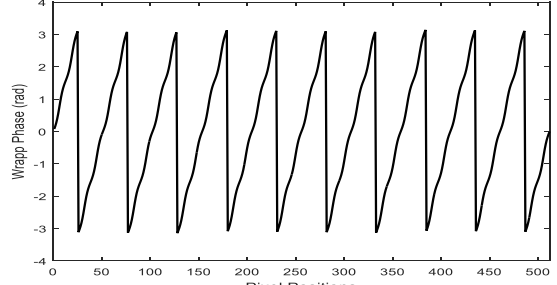

(d)

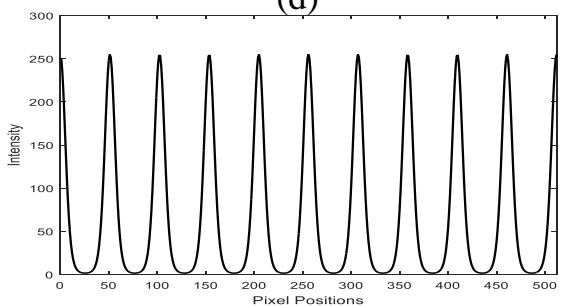

(f)

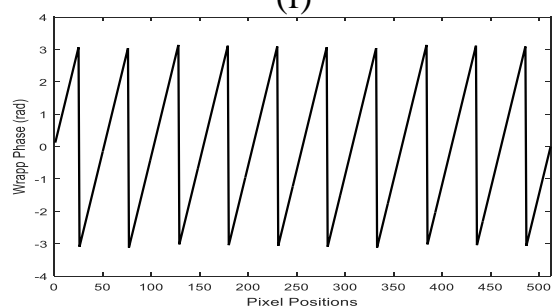

(h)

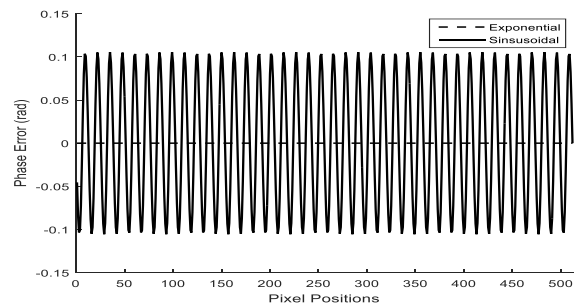

(j)

Figure 2 Simulation results obtained by four-step phase-shifting algorithm. (a)-(d) Results with traditional sinusoidal fringe patterns: (a) A captured fringe pattern; (b) profile from (a) along the middle row direction; (c) wrapped phase map; (d) profile from (c) along the middle row direction. (e)-(h) Results with the proposed exponential fringe patterns: (e) Captured fringe pattern; (f) profile from (e) along the middle row direction; (g) wrapped phase map; (h) profile from $(\mathrm{g})$ along the middle row direction. (i) profile of a part of absolute phase for two kinds of fringe patterns; (j) profile of phase errors with two kinds of fringe patterns.

For the proposed exponential fringe patterns, the corresponding wrapped phase map is not influenced by gamma distortion, resulting in a smooth wrapped phase distribution, as shown in Figure 2(g) and Figure 2(h). Figure 2(i) shows central cross-section line of a part of absolute phase for two kinds of fringe sequence. It is evident that the proposed exponential fringe projection method obtains a 
smooth absolute phase distribution while some waviness can be observed in the sinusoidal fringe projection method. The phase errors with two kinds of fringe patterns are obtained by subtracting the ideal phase value from the calculated phase value at each pixel point, as shown in Figure 2(j). The phase error with exponential fringe patterns is zero because they are independent of the gamma distortion. In contrast, the maximum value of phase error with sinusoidal fringe patterns is $0.1 \mathrm{rad}$ because they are severely influenced by gamma distortion.

In further simulations (Figure 3), different levels of random noise (0\% to $2.8 \%$ with an increment of $0.4 \%$ ) were added into the two kinds of phase-shifting fringe patterns for a range of $\gamma$ (between 2.2

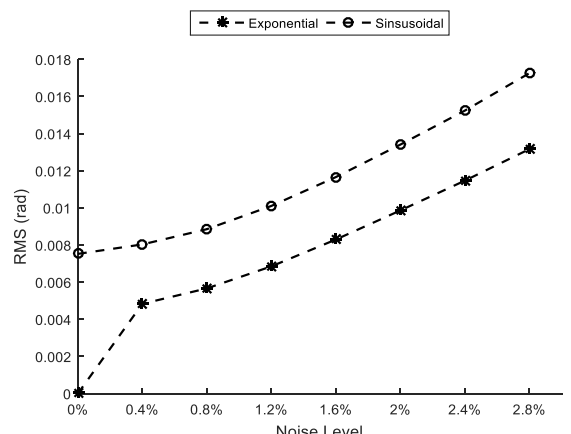

(a)

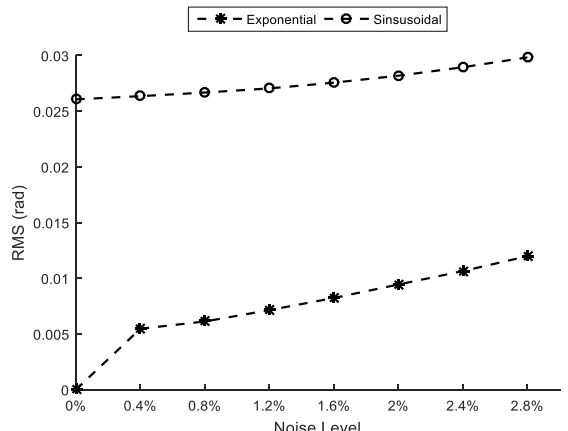

(c)

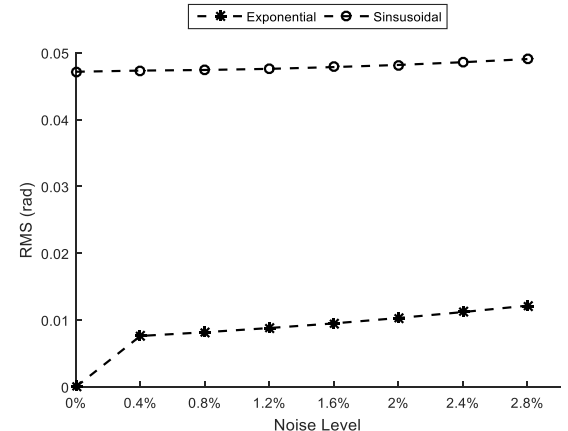

(e)

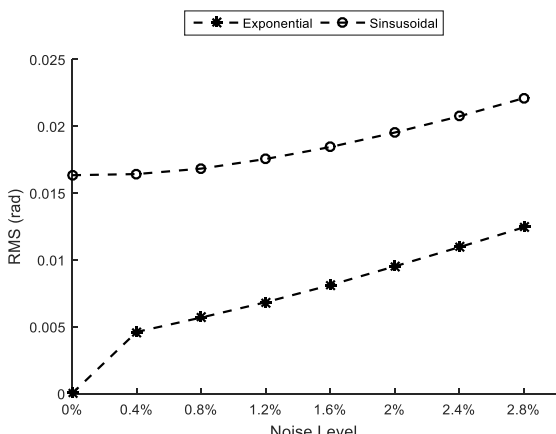

(b)

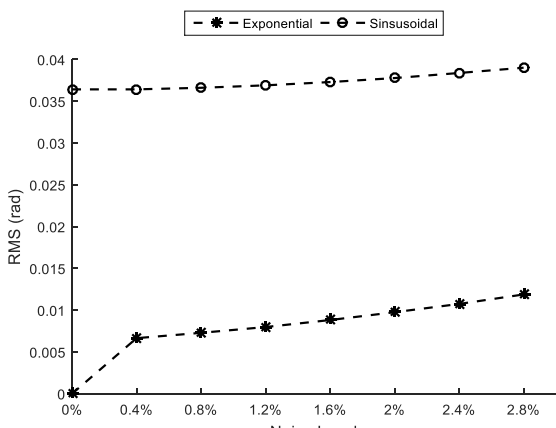

(d)

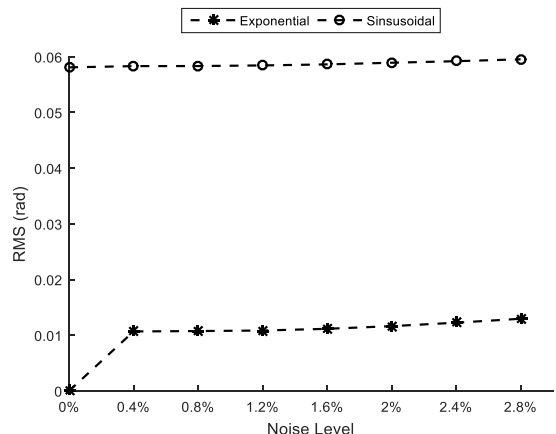

(f)

Figure 3 RMS errors of the phase for two kinds of fringe patterns. (a) - (f) RMS fringe patterns at $\gamma$ from 2.2 to 3.2 with an increment of 0.2 .

to 3.2 with an increment of 0.2). At $0 \%$ noise level, the RMS error for exponential fringe patterns is zero with $\gamma$ from 2.2 to 3.2, while there are RMS errors for sinusoidal fringe patterns. Although, the RMS errors for two kinds of fringe patterns increase with increasing $\gamma$, sinusoidal fringe patterns exhibit much larger errors as compared to exponential fringe patterns. This implies, that exponential fringe model can effectively alleviate phase error in PMP. 


\section{Experimental results}

A projector-camera arrangement was setup to verify the performance of the proposed method in a realistic measurement environment, as shown in Fig. 4. The setup includes a CCD camera (ECO655CVGE, SVS-VISTEK, Germany) and a digital light processing projector (CP270, BenQ). The projector resolution is $1024 \times 768$ pixels and the camera resolution is $2448 \times 2050$ pixels. A white plate was used as an imaging target for system calibration [21] and error analysis. For all experiments, a four-step phase-shifting algorithm ( $N=4$ in Eq. (3) and Eq. (12)) was used for wrapped phase calculation. Twelve fringe maps having the optimum fringe numbers [22] of 100, 99 and 90 were used for calculating absolute phase at each pixel position.

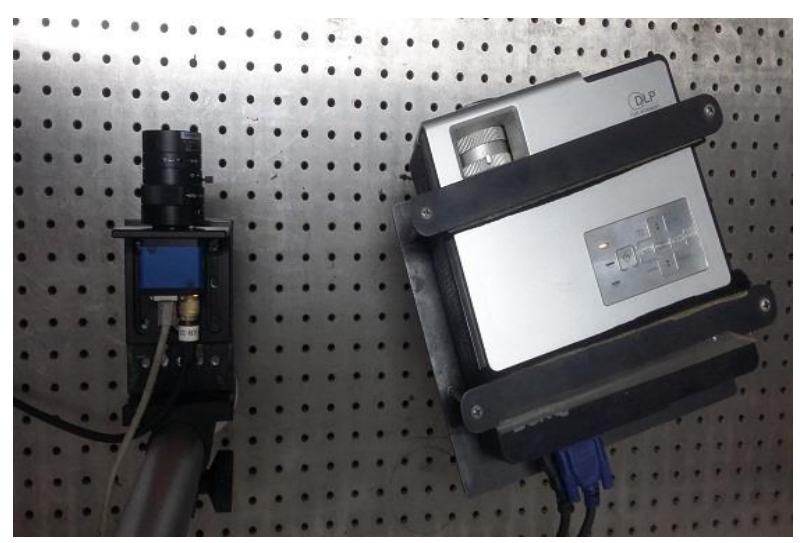

Figure 4 Three-dimensional measurement system based on fringe projection profilometry.

To evaluate the performance of the proposed method quantitatively, a plate was measured by using the traditional sinusoidal fringe projection method and the proposed gamma correction method. The plate was mounted on a precision stage and translated to 21 positions from $-30 \mathrm{~mm}$ to $30 \mathrm{~mm}$, in $3 \mathrm{~mm}$ increments. One position $(0 \mathrm{~mm})$ in the middle of the measuring volume was selected as a reference position. Exponential and traditional fringe patterns were generated by software according to Eq. (5) and Eq. (1) and projected onto surface of the measured objects by the projector. The CCD camera captured the two types deformed fringe pattern images from another viewpoint and the unwrapped phases were calculated by using Eq. (13) and Eq. (4), respectively. The phase errors of the plate with and without gamma correction at the reference position are shown in Fig. 5. It is evident that the phase error distribution with the proposed method (red line in Figure 5) is the lowest as compared with traditional method. The actual phase values of the plate in measuring volume are obtained from a 16-step phase-shifting algorithm according to Hoang and Pan's theory [8].

More detailed quantitative comparison results of the plate at the reference position are listed in Table 1, where MAX denotes the maximal phase error. The MAX and RMS phase error before gamma correction are $0.082 \mathrm{rad}$ and $0.029 \mathrm{rad}$, respectively. After using the proposed method, the corresponding errors decrease to $0.014 \mathrm{rad}$ and $0.0032 \mathrm{rad}$. The proposed method achieves a 9 fold reduction in the RMS of the phase errors. 


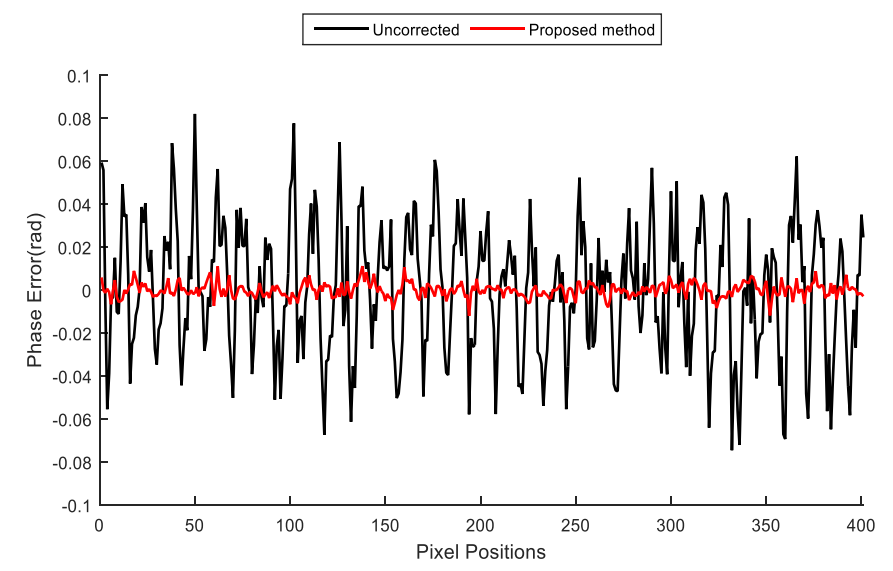

Figure 5 Profiles of phase errors along the middle row direction with and without the proposed gamma correction method.

Table 1. Quantitative comparison results of a flat plate (Unit: rad)

\begin{tabular}{lll}
\hline Method & Traditional & Proposed \\
\hline MAX & 0.082 & 0.014 \\
RMS & 0.029 & 0.0032
\end{tabular}

The RMS errors of the plate at each position are shown in Figure 6, where the error magnitude is 0.029-0.039 rad for the traditional method, while it is $0.0032-0.0034 \mathrm{rad}$ for the proposed method. It clearly shows that a 10 fold phase error can be achieved by the proposed method in a realistic measurement scenario.

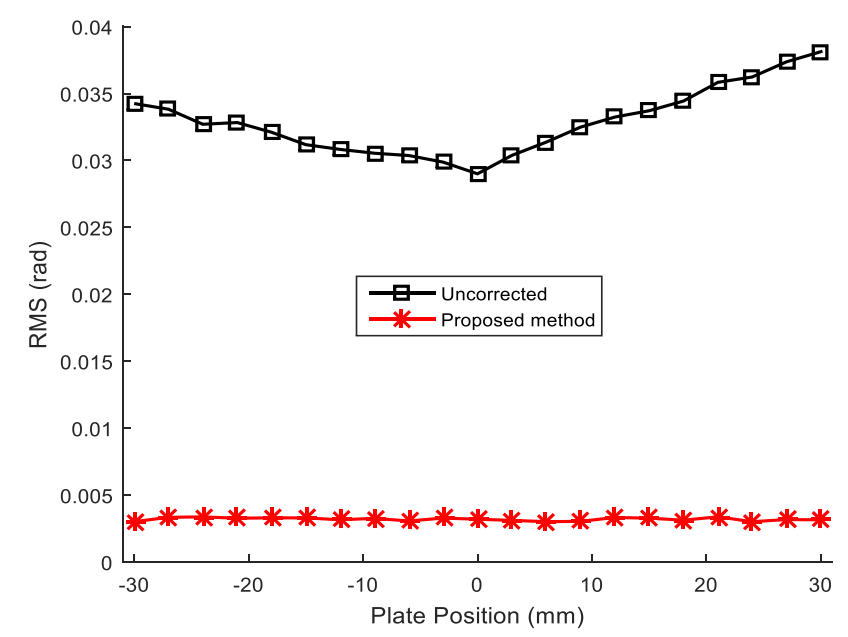

Figure 6 RMS errors over the measuring volume for a white plate

with and without the proposed gamma correction method.

To qualitatively evaluate the performance of the proposed method, a bear plaster model with a free form surface was measured by using the traditional and the proposed gamma correction methods. A typical reconstruction is shown in Figure 7(a) along with a profile (the black dashed line in Fig. 7(a)). It is evident that the reconstructed geometry (left of Figure 7(a)) without gamma correction contains a significant waviness due to phase errors. In contrast, the reconstructed geometry with the proposed method (Figure 7(b)) is smoother than the traditional method, indicating a drastic reduction in the phase error. The experimental results demonstrate that the proposed method can 
effectively reduce the phase error caused by the nonlinear response of the projector. Therefore, the proposed method provides a direct, simple yet effective solution to alleviate phase error, without involving gamma pre-calibration, iterative process or complicated computational cost.
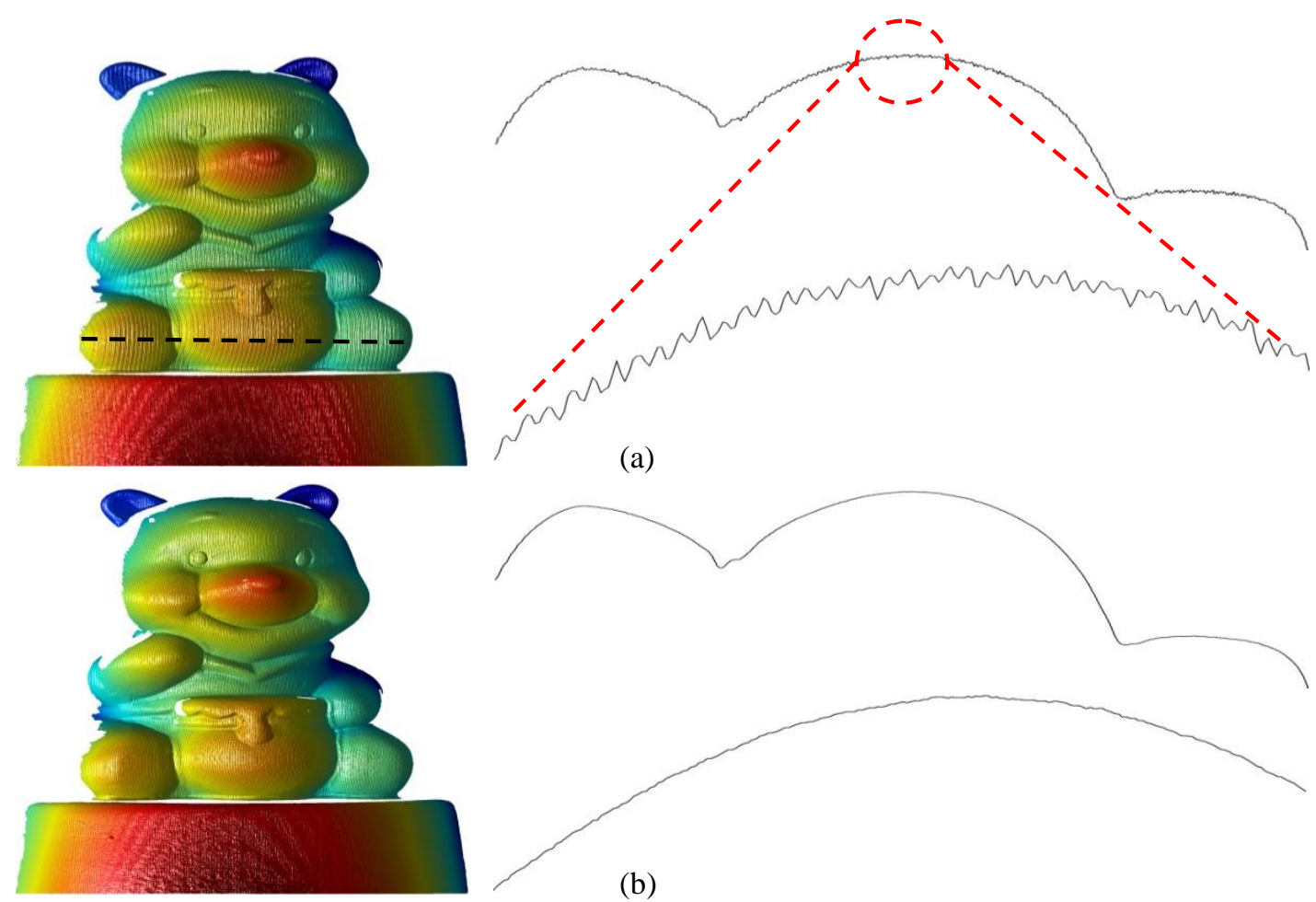

(b)

Figure 7 Reconstructed geometries and their profiles with and without the proposed gamma correction method for a bear plaster model.

In order to further generalize the proposed method, another projector (LightCrafter 4500, $912 \times$ 1140 pixels) was tested by measuring the same plate. A phase error distribution of the plate at the reference position along the middle row direction with and without the proposed gamma correction is shown in Fig. 8. The MAX and RMS values of the phase errors are summarized in Table 2. The proposed method achieves large reduction in error compared with the traditional phase computation without gamma correction.

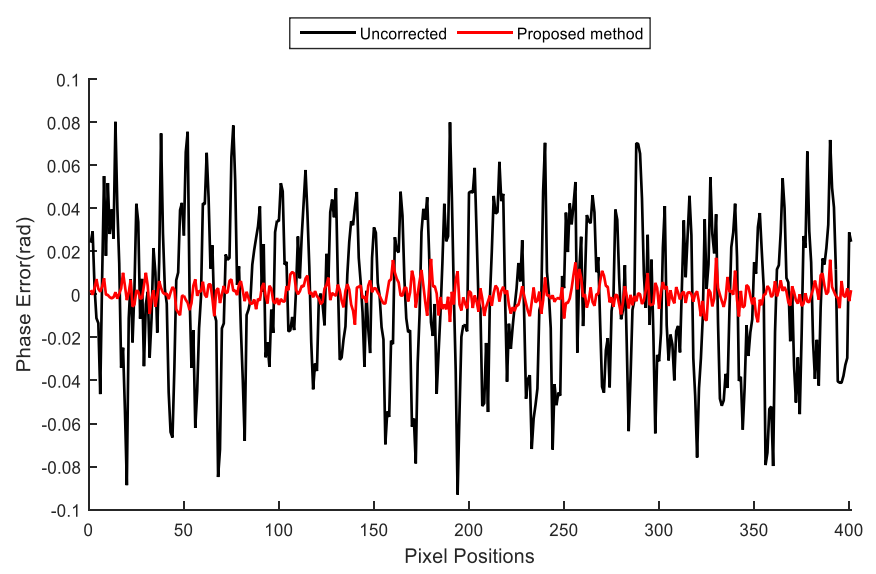

Figure 8 Profiles of phase errors along the middle row direction with and without the proposed gamma correction method. 
Table 2. Quantitative comparison results of LightCrafter 4500 projector model (Unit: rad)

\begin{tabular}{ccc}
\hline Method & Traditional & Proposed \\
\hline MAX & 0.093 & 0.012 \\
RMS & 0.017 & 0.0036 \\
\hline
\end{tabular}

\section{Conclusions}

A generic exponential fringe model has been derived from an optical perspective. Based on this model, a direct and simple gamma correction method is proposed for alleviating phase error. Compared with traditional sinusoidal fringe projection method, the proposed method decreases the phase error effectively and acquires higher quality measurement results. This work has three important contributions; first, the proposed exponential fringe model is suitable for any arbitrary multiple-step phase-shifting algorithm; second, the proposed gamma correction method can achieve a 10 fold phase error reduction compared to traditional method; third, the proposed method deals with the nonlinear problem at the source, instead of involving gamma pre-calibration, iterative processes or complex computational resources. Future work will involve the development of gamma correction methods that take into account the effect of ambient light and degree of defocusing of the fringe.

Acknowledgments: The authors would like to thank National Key R\&D Program of China (2017YFF0106404); National Natural Science Foundation of China (51675160); Key Basic Research Project of Applied Basic Research Programs Supported by Hebei Province (15961701D); Research Project for High-level Talents in Hebei University (GCC2014049); Talents Project Training Funds in Hebei Province (A201500503); Innovative and Entrepreneurial Talent Project Supported by Jiangsu Province, and Joint Doctoral Training Foundation of HEBUT(2017GN0002); European Horizon 2020 through the Marie Sklodowska-Curie Individual Fellowship Scheme (707466-3DRM); the UK's Engineering and Physical Sciences Research Council (EPSRC) funding of Future Advanced Metrology Hub (EP/P006930/1).

\section{References}

[1] Xu J, Liu S, Wan A, Gao B, Yi Q, Zhao D, Luo R, Chen C. An absolute phase technique for 3D profile measurement using four-step structured light pattern. Opt Laser Eng 2012; 50(9): 1274-1280.

[2] Zuo C, Huang L, Zhang M, Chen Q, Asundi A. Temporal phase unwrapping algorithm for fringe projection profilometry: a comparative review. Opt Laser Eng 2016; 85: 84-103.

[3] Zhou L, Zhou F, Qu D, Liu X, Lu W. Error analysis of the non-diffraction grating structured light generated by triangular prism. Opt Commun 2013; 306(10): 174-178.

[4] Cai Z, Liu X, Jiang H, He D, Peng X, Huang S, Zhang Z. Flexible phase error compensation based on Hilbert transform in phase shifting profilometry. Opt Express 2015; 23(19): 25171-25181.

[5] Huang P, Zhang S, Chiang C. High-speed 3-D shape measurement based on digital fringe projection. Opt Eng 2003; 42(1): 163-168.

[6] Zhang S, Yau S. Generic nonsinusoidal phase error correction for three-dimensional shape measurement using a digital video projector. Appl Opt 2007; 46(1): 36-43.

[7] Pan B, Qian K, Huang L, Asundi A. Phase error analysis and compensation for nonsinusoidal waveforms in phase-shifting digital fringe projection profilometry. Opt Lett 2009; 34(4): 416-418. 
[8] Hoang T, Pan B, Nguyen D, Wang Z. Generic gamma correction for accuracy enhancement in fringe-projection profilometry. Opt Lett 2010; 35(12): 1992-1994.

[9] Liu K, Wang Y, Lau D, Hao Q, Hassebrook L. Gamma model and its analysis for phase measuring profilometry. J Opt Soc Am A 2010; 29(6): 1047-1058.

[10] Li Z, Li Y. Gamma-distort fringe image modeling and accurate gamma correction for fast phase measuring profilometry. Opt Lett 2011; 36(2): 154-156.

[11]Zhang X, Zhu L, Li Y, Tu D. Generic nonsinusoidal fringe model and gamma calibration in phase measuring profilometry. J Opt Soc Am A 2012; 29(6): 1047-1058.

[12] Cai Z, Liu X, Peng X, Zhang Z, Jiang H, Yin Y, Huang S. Phase error compensation methods for high-accuracy profile measurement. Meas Sci Technol 2016; 27(4): 045201.

[13]Lei S, Zhang S. Flexible 3-D shape measurement using projector defocusing. Opt Lett 2009; 34(20): 3080-3082.

[14] Huang H, Fang X, Zhang W. Defocusing rectified multi-frequency patterns for high-precision 3D measurement. Meas Sci Technol 2014; 25(3), 35009-35022.

[15] Wang Y, Zhang, S. Optimal pulse width modulation for sinusoidal fringe generation with projector defocusing. Opt Lett 2010; 35(24): 4121-4123.

[16] Xu Y, Ekstrand L, Dai, J, Zhang S. Phase error compensation for three-dimensional shape measurement with projector defocusing. Appl Opt 2011; 50(17): 2572-2581.

[17]Zheng D, Da F. Absolute phase retrieval for defocused fringe projection three-dimensional measurement. Opt Commun 2014; 312(4), 302-311.

[18]Zeng Z, Fu Y, Li B, Chai M. Complex surface three-dimensional shape measurement method based on defocused gray plus phase-shifting. Opt Rev 2016; 23(4): 1-9.

[19] Wang Z, Nguyen D, Barnes J. Some practical considerations in fringe projection profilometry. Opt Laser Eng 2010; 48(2): 218-225.

[20] Liu Y, Xi Y, Yu Y, Chicharo J. Phase error correction based on inverse function shift estimation in phase shifting profilometry using a digital video projector. Proc SPIE 2010; 7855.

[21]Zhang Z, Ma H, Guo T, Zhang S, Chen J. Simple, flexible calibration of phase calculation-based three-dimensional imaging system. Opt. Lett. 2011; 36(7): 1257-1259.

[22]Zhang Z, Towers C, Tower D. Time efficient color fringe projection system for 3-D shape and color using optimum 3-frequency interferometry. Opt Express 2006; 14(14): 6444-6455. 